\title{
Quantitative analysis of exhaled carbonyl compounds distinguishes benign from malignant pulmonary disease
}

\author{
Michael Bousamra II, MD, ${ }^{a}$ Erin Schumer, MS, MD, ${ }^{a}$ Mingxiao Li, PhD, ${ }^{b}$ Ralph J. Knipp, MS, \\ Michael H. Nantz, PhD, ${ }^{\mathrm{c}}$ Victor van Berkel, $\mathrm{MD}, \mathrm{PhD},{ }^{\mathrm{a}}$ and Xiao-An Fu, $\mathrm{PhD}^{\mathrm{b}}$
}

\begin{abstract}
Objectives: The analysis of exhaled breath is a promising noninvasive tool for the diagnosis of lung cancer, but its clinical relevance has yet to be established. We report the analysis of exhaled volatile carbonyl compounds for the identification of specific carbonyl cancer markers to differentiate benign pulmonary disease from early-stage lung cancer and to compare its diagnostic accuracy with positron emission tomography (PET) scans.
\end{abstract}

\begin{abstract}
Methods: Aminooxy-coated silicon microchips were used for the selective capture of exhaled carbonyls by an oximation reaction. Breath samples were collected then directed through the silicon chips by applying a vacuum. Carbonyl adducts were analyzed by Fourier transform mass spectrometry. Eighty-eight control subjects, 107 patients with lung cancer (64 stage 0 , I, or II), 40 patients with benign pulmonary disease, and 7 patients with a solitary pulmonary metastasis participated. Analysis of cancer markers was performed blinded to the pathologic results.
\end{abstract}

Results: Four carbonyls were defined as cancer markers with significantly higher concentrations in patients with lung cancer. The number of increased cancer markers distinguished benign disease from both early and stage III and IV lung cancer. For early-stage disease, defining greater than 2 increased markers as diagnostic of lung cancer resulted in $83 \%$ sensitivity and $74 \%$ specificity. PET scans for this same cohort resulted in $90 \%$ sensitivity but only $39 \%$ specificity. Markers normalized for 3 of the 4 markers after resection of the lung cancer.

Conclusions: Analysis of specific exhaled carbonyls can differentiate early lung cancer from benign pulmonary disease. Breath analysis was more specific than PET for a lung cancer diagnosis. Judicious use of these data may expedite the care of patients with lung cancer. (J Thorac Cardiovasc Surg 2014;148:1074-81)

With the advent and increasing acceptance of computed tomography (CT) screening for lung cancer, the importance of distinguishing benign from malignant intrathoracic disease is increasing. The reported $20 \%$ reduction in lung cancer mortality from the National Lung Cancer Screening Trial is partially offset by the morbidity, cost and occasional mortality incurred by pursuing nonmalignant pulmonary nodules and adenopathy. ${ }^{1}$

Given a resectable pulmonary nodule, and depending on its degree of suspicion, there are multiple options for diagnostic workup. ${ }^{2}$ Serial CT scanning to observe growth, resolution, or stability is commonly used for subcentimeter lesions. Positron emission tomography (PET) scintigraphy

From the Departments of Cardiovascular and Thoracic Surgery, ${ }^{\mathrm{a}}$ Chemical Engineering, ${ }^{\mathrm{b}}$ and Chemistry, ${ }^{\mathrm{c}}$ University of Louisville, Louisville, Ky.

This work was partially supported by the Clinical and Translational Science Pilot Grant Program (CTSPGP 20035) of the University of Louisville and the National Science Foundation (CBET:1159829).

Disclosures: Authors have nothing to disclose with regard to commercial support.

Read at the 94th Annual Meeting of The American Association for Thoracic Surgery, Toronto, Ontario, Canada, April 26-30, 2014.

Received for publication April 9, 2014; revisions received May 27, 2014; accepted for publication June 4, 2014.

Address for reprints: Michael Bousamra II, MD, Department of Cardiovascular and Thoracic Surgery, University of Louisville, 201 Abraham Flexner Way, Suite 1200, Louisville, KY 40202 (E-mail: mbousamra@louisvilleheartsurgery.com).

$0022-5223 / \$ 36.00$

Copyright (c) 2014 by The American Association for Thoracic Surgery

http://dx.doi.org/10.1016/j.jtcvs.2014.06.006 of the lesion may be used to assess the probability of malignancy in nodules larger than $8 \mathrm{~mm}$. Bronchoscopy and percutaneous biopsy are options for tissue acquisition but the associated costs and risks are significant. In addition, diagnostic yields are highly conditional with respect to tumor size, location, and operator skill. Proceeding directly to surgical resection is appropriate when the probability of malignancy is high and the surgical risk is low. Conversely, surgical resection of nonmalignant disease is a clinical failure because, in most such instances, the natural course of the benign process would never have harmed the patient. Furthermore, PET scans are frequently falsely positive both in cases of solitary pulmonary nodules and in cases with hilar and mediastinal adenopathy. All may lead to increased clinical suspicion of lung cancer and an obligation to rule out malignancy by surgical intervention. Thus, it is important to develop reliable methods that minimize the diagnostic burden to patients who have no significant disease while expediting treatment in patients who have lung cancer. $^{2-4}$

Breath analysis of patients with suspected lung cancer is a developing modality with the potential to fulfill this goal. ${ }^{5}$ Linus Pauling first studied the volatile constituents of breath in individuals maintained on an elemental diet in $1971 .^{6}$ In recent years, the analysis of exhaled breath has become a broad research frontier. ${ }^{5,7,8}$ Several approaches have been developed, including sensor arrays, ${ }^{9-13}$ proton transfer 

Abbreviations and Acronyms
$\mathrm{ATM}=2$-(aminooxy)- $N, N, N$ -
trimethylethanammonium
$\mathrm{CT}=$ computed tomography
$\mathrm{ECM}=$ elevated cancer marker
FT $=$ Fourier transform
$\mathrm{GC}=$ gas chromatography
$\mathrm{ICR}=$ ion cyclotron resonance
MS = mass spectrometry
PET $=$ positron emission tomography
$\mathrm{SUV}=$ standardized uptake value

reaction mass spectrometry, ${ }^{14,15}$ selected ion flow tube mass spectrometry, ${ }^{16,17}$ and gas chromatography-mass spectrometry. ${ }^{18-23}$

Reports of breath analysis of patients who have lung cancer have indicated a host of associated compounds and profiles ${ }^{5,19,24}$; however, the diagnostic usefulness of breath analysis has not been established by these results. The composition of breath ranges from molecular hydrogen to more than 1000 volatile organic compounds and nonvolatile condensates. ${ }^{5,7,25,26}$ When analyzing such a broad data set, the potential for false leads and confounding factors increases. The method described here focuses selectively on aldehydes and ketones, volatile carbonyl compounds in breath. This approach was chosen because increased levels of carbonyls have been reported in exhaled breath of patients with lung cancer. ${ }^{18-22}$ Recently, we reported the identification of 4 carbonyl markers that distinguish lung cancer from benign disease, lung cancer histology, and non-small lung cancer stages. ${ }^{27}$ This report focuses on the clinical usefulness of this diagnostic method in differentiating early-stage lung cancer from benign pulmonary disease. The diagnostic accuracy of breath analysis is compared with the PET standardized uptake value (SUV). To the best of our knowledge, this is the first comparison of a breath analysis diagnostic method with PET SUV results. We also report that a variety of cancers metastatic to the lung produce increased levels of the same carbonyl markers. We also show that the increased carbonyl markers return to the level of healthy controls after resection of malignancy.

\section{MATERIALS AND METHODS}

\section{The Silicone Microreactor Chips}

The microreactor chips were fabricated from $10-\mathrm{cm}$ silicon chips using previously published microelectromechanical systems fabrication techniques. ${ }^{28,29}$ The size of the silicon chip is similar to the size of a dime and consists of an array of thousands of micropillars in the microfluidic channel to uniformly distribute gas flowing through the channel. A quaternary ammonium compound, 2-(aminooxy)- $N, N, N$ trimethylethanammonium (ATM) iodide was used to coat the surfaces of the micropillars as previously described. ${ }^{28,29}$ ATM adsorbs to the silicon dioxide surfaces of the micropillars via electrostatic and hydrogen bond interactions. ATM chemoselectively traps carbonyl compounds in exhaled breath by means of oximation reactions. Capture efficiencies of $98 \%$ or more have been verified for carbonyl compounds. ${ }^{29}$

\section{Fourier Transform-Ion Cyclotron Resonance-Mass Spectrometry}

A hybrid linear ion trap Fourier transform (FT)-ion cyclotron resonance (ICR)-mass spectrometer (MS) (Finnigan LTQ FT, Thermo Electron, Bremen, Germany) equipped with a TriVersaNanoMate ion source (AdvionBioSciences, Ithaca, NY) with an electrospray chip (nozzle inner diameter $5.5 \mu \mathrm{m}$ ) was used to analyze all breath samples. The TriVersaNanoMate was operated in positive ion mode by applying $2.0 \mathrm{kV}$ with no head pressure. ATM and its adducts are cations that do not need further ionization. These uniformly charged molecules are an important advantage of using ATM for capture of carbonyl compounds and for analysis by FT-ICR-MS. ${ }^{29}$ The detailed procedure for analysis by FT-ICR-MS has been previously delineated. ${ }^{29}$

\section{Collection of Breath Samples}

The detailed research protocol for the collection of exhaled breath samples was approved by the Institutional Review Board at the University of Louisville. All study participants signed informed consent before providing breath samples. One liter of breath was collected into a Tedlar bag (SigmaAldrich, St Louis, Mo) from a single exhalation from each participant. Breath samples were first collected from 88 healthy controls; 45 were current smokers and 43 were never smokers. A total of 171 patients with suspected or confirmed cancer of the lung participated. Primary lung cancer was present in 107 patients (97 non-small cell lung cancer, 8 small cell lung cancer, 1 combined small and non-small cell lung cancer, and 1 carcinoid tumor), and 40 patients had benign pulmonary disease (single or multiple pulmonary nodules and/or mediastinal adenopathy). Age and smoking information for the 3 groups is summarized in Table 1. Healthy controls and patients with benign pulmonary disease were younger than the patients with lung cancer. Smoking history was similar. Seven additional patients had a solitary metastasis to the lung. Five patients with presumptive lung cancer were treated with radiation therapy without tissue confirmation and were excluded from the analysis. Twelve patients were excluded because a diagnosis had not yet been reached.

The procedure for the capture of carbonyl compounds in air and exhaled breath has been described previously. ${ }^{28,29}$ In brief, the exhaled breath collected in 1-L Tedlar bags was drawn through the microreactor chip by applying a vacuum. After this process, ATM adducts in the microreactor chip were eluted with $100 \mu \mathrm{L}$ of methanol from a slightly pressurized small vial. Ninety-nine percent of ATM adducts were recovered. The eluted solution was analyzed directly by FT-ICR-MS. A known amount of deuterated acetone completely reacted with ATM (ATM-acetone- $\mathrm{d}_{6}$ ) in methanol was added to the eluted solution as an internal reference. The concentrations of all carbonyl compounds in exhaled breath were determined by comparison of the relative abundance with that of added ATM-acetone- $\mathrm{d}_{6}$.

\section{Data Analysis}

As previously described, analysis of carbonyl concentrations in breath samples of patients was performed with 88 healthy controls followed by the first 10 confirmed cases of lung cancer. Four carbonyl cancer markers were identified as being higher than healthy control levels and served as presumptive cancer markers in all subsequent analyses between healthy controls and patient subsets. ${ }^{27}$ Carbonyl concentrations for all exhaled breath samples were analyzed by the Wilcoxon test to determine statistically significant differences between groups. Because the concentrations of the carbonyl compounds detected had a nonnormal distribution, the Wilcoxon test was used instead of analysis of variance. The Wilcoxon tests were performed using Minitab version 16.0 (Mimitab, Inc, State College, Pa). 
TABLE 1. Description of the patients

\begin{tabular}{|c|c|c|c|c|c|}
\hline \multirow[b]{2}{*}{ Subjects } & \multirow[b]{2}{*}{ Total no. } & \multirow{2}{*}{$\begin{array}{c}\text { Average age, } \\
\text { y } \pm \text { standard } \\
\text { deviation }\end{array}$} & \multicolumn{3}{|c|}{ Smoking history } \\
\hline & & & Current & Former & Never \\
\hline Healthy controls & 88 & $42.2 \pm 14.2$ & 45 & 0 & 43 \\
\hline $\begin{array}{l}\text { Benign pulmonary } \\
\text { disease }\end{array}$ & 40 & $50.9 \pm 14.5$ & 12 & 14 & 14 \\
\hline Lung cancer & 107 & $65.8 \pm 10.1$ & 44 & 51 & 12 \\
\hline
\end{tabular}

\section{RESULTS}

Four carbonyl compounds, 2-butanone $\left(\mathrm{C}_{4} \mathrm{H}_{8} \mathrm{O}_{2}\right)$ $(P<.0001)$, 3-hydroxy-2-butanone $\left(\mathrm{C}_{4} \mathrm{H}_{8} \mathrm{O}_{2}\right)(P<.0001)$, 2-hydroxyacetaldehyde $\left(\mathrm{C}_{2} \mathrm{H}_{4} \mathrm{O}_{2}\right) \quad(P<.0001)$, and 4-hydroxyhexenal $\left(\mathrm{C}_{6} \mathrm{H}_{10} \mathrm{O}_{2}\right)(P=.0045)$ were identified as significantly increased in patients with lung cancer compared with the healthy control group. ${ }^{27}$ The carbonyl marker concentrations in the healthy control, benign pulmonary disease, and lung cancer groups are presented in Figure 1. Although the median cancer marker concentrations of patients with lung cancer are significantly higher than that of both healthy controls and patients with benign disease, there is overlap between the concentration ranges of the cancer markers between the groups. Thus, no single cancer marker was sufficient to differentiate malignant from benign disease. This finding prompted us to define an elevated cancer marker (ECM) concentration as one that exceeds the range of the healthy control distribution. Therefore, a given patient may have 0 to 4 ECMs.

Table 2 presents a matrix relating the number of ECMs to lung cancer stage and patients with benign pulmonary disease. The matrix coefficients represent the number of patients in each disease set with a given number of ECMs. Ninety-four of 107 patients with lung cancer have 2 or more ECMs. Thirty-one of 40 patients with benign pulmonary disease have less than 2 ECMs. Only 1 patient with stage III cancer demonstrated less than 2 ECMs. A logistic regression model consisting of actual concentrations of volatile compounds showed that $\mathrm{C}_{2} \mathrm{H}_{4} \mathrm{O}_{2}$ (odds ratio, 44.4; $P<.05$ ) was an independent predictor of cancer. A receiver operating curve based on the logistic regression model showed $86 \%$ area under the curve.

Sixty-four patients were identified with early-stage lung cancer (stage 0, I, or II) (Table 2). Fifty-two $(81.3 \%$ ) of these patients had 2 or more ECMs. Eleven patients with lung cancer had less than 2 ECMs. Ten were stage IA or 0 . Nine had an adenocarcinoma and 1 had squamous cell carcinoma. The remaining false-negative patient had a lymphocyte-rich sarcomatoid cancer and was stage IIB.

The sensitivity and specificity of breath analysis varies according to the number of ECMs that define a positive result. Comparing patients with early-stage lung cancer with those with benign disease, if 3 or more ECMs are chosen as a predictor of cancer, then a sensitivity of $55 \%$ and a specificity of $95 \%$ is achieved. If 2 or more ECMs are chosen, the sensitivity increases to $81 \%$ and the specificity decreases to $74 \%$. Likewise, if 1 or more ECM is chosen, the sensitivity is $95 \%$ and the specificity is $45 \%$. When applying breath analysis to tumors of $2.5 \mathrm{~cm}$ or smaller, the sensitivity and specificity for 2 or more ECMs were $74 \%$ (28 of 38 ) and $81 \%$ (17 of 21 ), respectively, and for 3 or more ECMs, the sensitivity
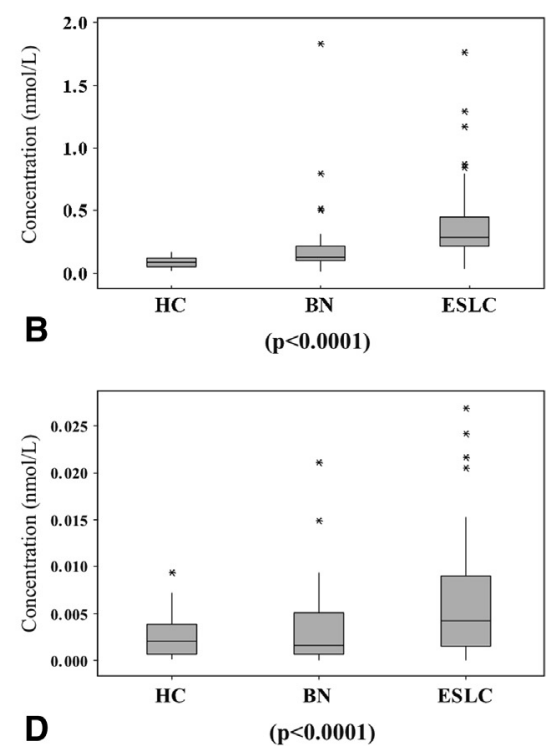

FIGURE 1. The box plots of the 4 cancer markers for the healthy control, benign pulmonary disease patient and lung cancer patient groups. A, 2-Butanone; B, 3-hydroxy-2-butanone; C, 2-acetacetaldehyde; D, 4-hydroxyhexenal. $P$ values apply to the benign versus lung cancer patient groups. The box plot presents the median, lower, and upper quartiles (25th to 75 th percentiles). $H C$, Healthy control group; $B N$, benign pulmonary patient group; $L C$, lung cancer patient group; ESLC, early-stage lung cancer; 4-HHE, 4-hydroxyhexenal. 
TABLE 2. Sensitivity and specificity based on the number of elevated cancer markers in lung cancer and benign pulmonary disease

\begin{tabular}{|c|c|c|c|c|c|}
\hline \multirow[b]{2}{*}{ Patients } & \multicolumn{5}{|c|}{ Elevated cancer markers } \\
\hline & 4 & 3 & 2 & 1 & $\mathbf{0}$ \\
\hline Early stage, stage 0, I, II (n) & 14 & 21 & 17 & 7 & 5 \\
\hline Sensitivity (\%) & 21 & 55 & 81 & 92 & \\
\hline Specificity (\%) & 100 & 95 & 74 & 45 & \\
\hline All-stage cancer (n) & 30 & 42 & 22 & 8 & 5 \\
\hline Sensitivity (\%) & 28 & 67 & 88 & 95 & \\
\hline Specificity (\%) & 100 & 95 & 77 & 45 & \\
\hline Benign pulmonary nodule & 0 & 2 & 7 & 13 & 18 \\
\hline
\end{tabular}

Sensitivity and specificity based on cumulatively positive elevated cancer markers; eg, sensitivity under the column headed 3 means sensitivity for 3 or more ECMs.

and specificity were 50\% (19 of 38) and 95\% (20 of 21 ), respectively (Figure 2). For tumors greater than $2.5 \mathrm{~cm}$, breath analysis was always positive with 2 or more ECMs.

Among patients with benign pulmonary nodules, 27\% had multiple nodules and adenopathy, $27 \%$ had a solitary nodule, $22 \%$ had multiple nodules, $17 \%$ had a solitary nodule and adenopathy, and 5\% had adenopathy. The mean size of the largest pulmonary nodule for a given patient was $2.1 \pm 1.2 \mathrm{~cm}$. A tissue diagnosis was obtained in $29(73 \%)$ patients by bronchoscopy, CT-guided biopsy, or surgical biopsy. Of these patients, $15(50 \%)$ had a granulomatous process, $11(36.7 \%)$ had inflammatory cells, and $3(10.0 \%)$ had pneumonia. Those patients who did not undergo a biopsy had low clinical suspicion for cancer and were followed with serial CT scans.

A PET scan was performed in $93(87 \%)$ of the patients with lung cancer and $31(78 \%)$ of the patients with benign pulmonary disease. For the entire cohort, the sensitivity and specificity using PET scans were $92.5 \%$ and $38.7 \%$, respectively, compared with $88 \%(P=.4)$ and $77.0 \%$ $(P=.004)$, respectively, for breath analysis when ECMs were set at 2 or more. Comparing early-stage lung cancer $(\mathrm{n}=64)$ and benign pulmonary disease, the diagnostic sensitivity and specificity of PET scans were $90.3 \%$ and $38.7 \%$, respectively. The sensitivity and specificity of breath analysis for these patients were $81 \%(P=.3)$ and $74.0 \%(P=.002)$, respectively. Thus, PET scans and breath analysis had similar sensitivity; however, the specificity of breath analysis was nearly double that of PET scan. Focusing on 37 patients with stage 0 and I tumors that were less than $3.0 \mathrm{~cm}$ yields similar results. Breath analysis was correctly diagnostic of lung cancer in this group for 27 patients, resulting in a sensitivity of $73.0 \%$. PET scan accurately diagnosed lung cancer in 30 patients, resulting in a similar sensitivity of $81 \%$.

Seven patients had isolated metastases to the lung. They also demonstrated ECMs in proportions similar to patients with lung cancer. Primary sites included the colon $(\mathrm{n}=3$; 3, 2, 1 ECMs), skin melanoma ( $n=2 ; 3,1 \mathrm{ECMs})$, prostate $(\mathrm{n}=1 ; 4 \mathrm{ECMs})$, and breast $(\mathrm{n}=1 ; 3 \mathrm{ECMs})$.

Breath was also collected from 20 patients with early-stage lung cancer at least 1 month after complete resection. Figure 3 shows box plots of the 4 cancer markers identified. The concentrations of 2-butanone, 2-hydroxyacetaldehyde, and 4-hydroxyhexenal are significantly reduced after resection, however the reduction in 3-hydroxy-2-butanone concentration did not achieve statistical significance.

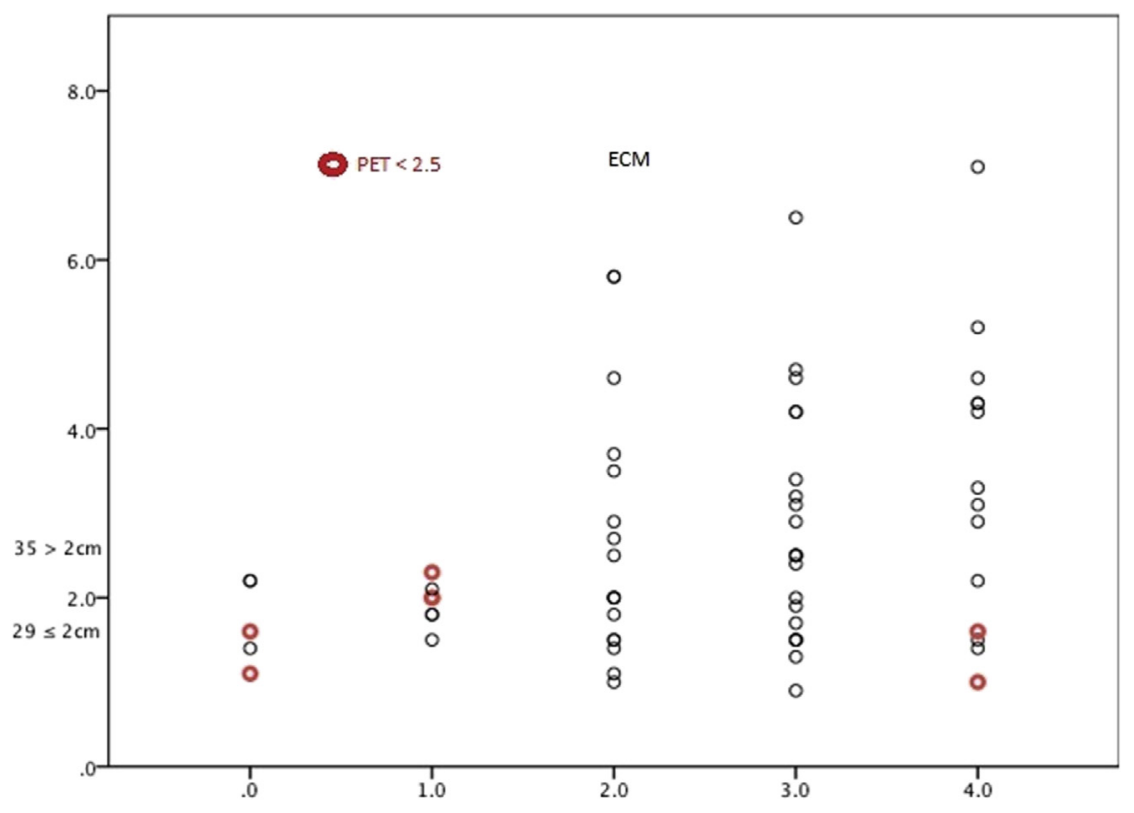

FIGURE 2. Elevated carbonyl markers $(E C M s)$ and positron emission tomography $(P E T)$ results in early-stage lung cancer. False-negative PET scans $(\mathrm{n}=6)$ are identified by red circles. All tumors $>2.5 \mathrm{~cm}$ have $\geq 2$ ECMs, 35 tumors are $>2 \mathrm{~cm}$ and 19 are $\leq 2 \mathrm{~cm}$. 

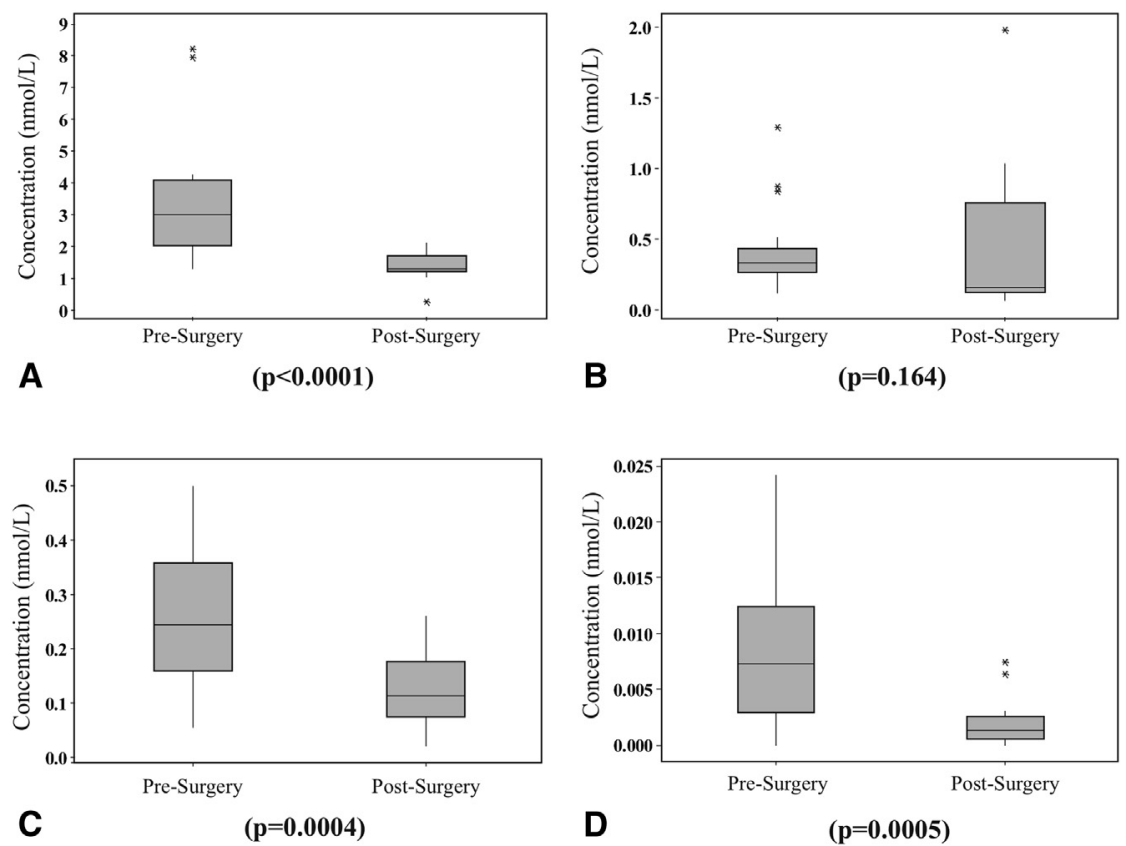

FIGURE 3. Comparison of box plots of the 4 carbonyl markers before and after early-stage cancer resection. The box plot presents the median, lower, and upper quartiles (25th to 75th percentiles). A, 2-Butanone, $P=.0001 ; \mathrm{B}, 3$-hydroxy-2-butanone, $P=.164$; C, 2-acetaldehyde, $P=.0004$; $\mathrm{D}$, 4-hydroxyhexenal, $P=.0005$. 4-HHE, 4-hydroxyhexenal.

\section{DISCUSSION}

Exhaled breath analysis of patients with lung cancer identified 4 increased carbonyl compounds that served as cancer markers. Among patients with lung cancer, 3 or 4 ECMs produced a specificity of $95 \%$. Furthermore, $60 \%$ of patients with early-stage lung cancer had 3 or more ECMs. A positive breath result with 3 or 4 ECMs could direct care to surgical resection as the next step for patients with low suspicion of metastasis. Conversely, breath analysis rarely yields a false-negative result in advanced stages of lung cancer and has a much lower false-positive rate for benign disease compared with PET scans. Thus, if the pretest probability of benign disease is high, performing a breath analysis as the next study rather than a PET scan may save patients from invasive biopsy procedures.

Pulmonary nodules associated with a negative breath analysis result may still require biopsy or repeated imaging depending on their degree of suspicion because $24 \%$ of patients with stage 0 and stage I lung cancer had fewer than 2 ECMs. One patient with stage III and 1 with stage II lung cancer had 1 ECM and no ECMs, respectively. As a corollary, it seems unlikely that this breath analysis method could supplant CT as a primary screening tool because of the significant false-negative rate. Its primary potential is in conjunction with a positive result on a CT scan. It remains to be determined what proportion of subcentimeter malignant pulmonary nodules will be diagnosed by combined CT screening and breath analysis.
There are numerous reports of breath analysis methods for lung cancer detection. ${ }^{6,9-22,30-32}$ Most have involved complicated concentration techniques and data analysis, making clinical application remote. The current method is simple for the patient and processing and analysis are straightforward. FT-ICRS-MS analysis is direct with unequivocal identification of molecular formulae by the exact mass observed. Isomers of a given molecular formula may require further analytical separation and identification using gas chromatography(GC)-MS or liquid chromatographyMS/MS. This technique is also highly precise. Carbonyl compounds were detected over a wide range of concentrations by FT-ICR-MS, from $8 \mathrm{nmol} / \mathrm{L}$ for 2-butanone to $7 \times 10^{-5} \mathrm{nmol} / \mathrm{L}$ for 4-hydroxyhexenal. ${ }^{27}$ Furthermore, the carbonyl mass spectra provide a library of hard data that can be retrieved and reanalyzed as new markers are postulated.

Recent publications referencing a gold, nanoparticlebased, gas sensor nanoarray detection device are enticing. In a smaller sample set than in the current study, Peled et al. ${ }^{10}$ were able to distinguish benign from malignant disease and early from advanced disease, however a specific comparison of early lung cancer with benign disease was not given. A similar gas sensor nanoarray study in conjunction with GC-MS followed patients postoperatively and demonstrated that the levels of specific volatile compounds were increased when cancer was present and returned to normal levels postoperatively. ${ }^{30}$ 
For the future, defining the pretest probability of lung cancer before diagnostic breath analysis would be incisive in determining the added benefit of our breath analysis method. PET scans in patients with bulky disease frequently result in false-positives for truly benign conditions as demonstrated by the low specificity of PET scans. This breath analysis method provides the potential for a cheaper and more reliable diagnostic option for patients found to have bulky disease on CT scans. If negative by breath analysis, the patient may be followed clinically without tissue diagnosis. If positive by breath analysis, then the patient may proceed to definitive biopsy, thus expediting the treatment of lung cancer.

Our study has several limitations. Control subjects were younger and healthy with no known active pulmonary disease, which may represent a bias. The proportion of patients with benign disease is low and further studies that specifically compare a larger number of patients with solitary pulmonary nodules and lung cancer is warranted. A search for disease processes that may produce the same carbonyl cancer markers is ongoing. Preliminary data among patients with cystic fibrosis and pulmonary fibrosis has not indicated any interference of carbonyl markers. Environmental and genetic factors can be addressed by studying relatives of patients with cancer. Age-matched controls and specific studies on patients with chronic obstructive pulmonary disease are warranted.

The significance of our data is reinforced by the normalization of 3 of the 4 cancer markers within 1 month of cancer resection. Postoperative studies need to be extended to all patients after resection with serial follow-up to determine if breath analysis can serve as a marker for tumor recurrence. This study also needs to be extended to cancers of different origin to determine applicability beyond lung cancer. The finding that metastatic disease to the lung produced increased levels of carbonyl markers is enticing, and promotes the concept that these markers may be found in other primary tumors. A larger data set is needed to further refine our results and compare its diagnostic usefulness with screening $\mathrm{CT}$ scans. By resetting the cancer marker concentration parameters to ensure maximum sensitivity, a prospective study comparing screening CT scans and breath analysis could be performed.

\section{CONCLUSIONS}

The increased concentrations of 2-butanone, 3-hydroxy2-butanone, 2-hydroxyacetaldehyde, and 4-hydroxyhexenal in exhaled breath can be used for the diagnosis of lung cancer. The sensitivity and specificity of breath analysis are related to the number of the ECMs. Breath analysis has similar sensitivity to PET for the diagnosis of lung cancer, but has much higher specificity than PET in distinguishing benign disease. Patients with lung metastases also demonstrated ECMs. After resection of lung cancer, 3 of
4 cancer markers decreased to healthy control range. The judicious use of these findings may expedite the care and influence decision-making in patients with potential lung cancer.

\section{References}

1. National Lung Screening Trial Research Team, Aberle DR, Adams AM, Berg CD, Black WC, Clapp JD, et al. Reduced lung-cancer mortality with low-dose computed tomographic screening. N Engl J Med. 2011;365:395-409.

2. Van't Westeinde SC, van Klaveren RJ. Screening and early detection of lung cancer. Cancer J. 2011;17:3-10.

3. Savage N. Early detection: spotting the first signs. Nature. 2011;471:S14-5.

4. Early warnings. Nature. 2009;458:679.

5. Hakim M, Broza YY, Barash O, Peled N, Phillips M, Amann A, et al. Volatile organic compounds of lung cancer and possible biochemical pathways. Chem Rev. 2012;112:5949-66.

6. Pauling L, Robinson AB, Teranishi R, Cary P. Quantitative analysis of urine vapor and breath by gas-liquid partition chromatography. Proc Natl Acad Sci U SA. 1971;68:2374-6.

7. Zhang ZM, Ma YJ, Li GK. Progress on the analytical methodology for biological volatile organic compounds. Anal Methods. 2013;5:20-9.

8. Spanel P, Smith D. Volatile compounds in health and disease. Curr Opin Clin Nutr Metab Care. 2011;14:455-60.

9. Mazzone PJ, Wang XF, Xu Y, Mekhail T, Beukemann MC, Na J, et al. Exhaled breath analysis with a colorimetric sensor array for the identification and characterization of lung cancer. J Thorac Oncol. 2012;7:137-42.

10. Peled N, Hakim M, Bunn PA Jr, Miller YE, Kennedy TC, Mattei J, et al. Noninvasive breath analysis of pulmonary nodules. J Thorac Oncol. 2012;7:1528-33.

11. Peng G, Hakim M, Broza YY, Billan S, Abdah-Bortnyak R, Kuten A, et al. Detection of lung, breast, colorectal, and prostate cancers from exhaled breath using a single array of nanosensors. Br J Cancer. 2010;103:542-51.

12. Peng G, Tisch U, Adams O, Hakim M, Shehada N, Broza YY, et al. Diagnosing lung cancer in exhaled breath using gold nanoparticles. Nat Nanotechnol. 2009;4 669-73.

13. Hakim M, Billan S, Tisch U, Peng G, Dvrokind I, Marom O, et al. Diagnosis of head-and-neck cancer from exhaled breath. Br J Cancer. 2011;104:1649-55.

14. Schwarz K, Filipiak W, Amann A. Determining concentration patterns of volatile compounds in exhaled breath by PTR-MS. J Breath Res. 2009;3:027002.

15. Brunner C, Szymczak W, Hollriegl V, Mortl S, Oelmez H, Bergner A, et al. Discrimination of cancerous and non-cancerous cell lines by headspaceanalysis with PTR-MS. Anal Bioanal Chem. 2010;397:2315-24.

16. Spanel P, Smith D. Progress in SIFT-MS: breath analysis and other applications. Mass Spectrom Rev. 2011;30:236-67.

17. Cap P, Dryahina K, Pehal F, Spanel P. Selected ion flow tube mass spectrometry of exhaled breath condensate headspace. Rapid Commun Mass Spectrom. 2008; 22:2844-50

18. Song G, Qin T, Liu H, Xu GB, Pan YY, Xiong FX, et al. Quantitative breath analysis of volatile organic compounds of lung cancer patients. Lung Cancer. 2010; 67:227-31.

19. Bajtarevic A, Ager C, Pienz M, Klieber M, Schwarz K, Ligor M, et al Noninvasive detection of lung cancer by analysis of exhaled breath. BMC Cancer. 2009:9:348.

20. Fuchs P, Loeseken C, Schubert JK, Miekisch W. Breath gas aldehydes as biomarkers of lung cancer. Int J Cancer. 2010;126:2663-70.

21. Poli D, Goldoni M, Corradi M, Acampa O, Carbognani P, Internullo E, et al. Determination of aldehydes in exhaled breath of patients with lung cancer by means of on-fiber-derivatisation SPME-GC/MS. J Chromatogr B Analyt Technol Biomed Life Sci. 2010;878:2643-51.

22. Phillips M, Altorki N, Austin JH, Cameron RB, Cataneo RN, Kloss R, et al Detection of lung cancer using weighted digital analysis of breath biomarkers. Clin Chim Acta. 2008:393:76-84.

23. Ligor T, Ligor M, Amann A, Ager C, Bachler M, Dzien A, et al. The analysis of healthy volunteers' exhaled breath by the use of solid-phase microextraction and GC-MS. J Breath Res. 2008;2:046006.

24. Chan HP, Lewis C, Thomas PS. Oxidative stress and exhaled breath analysis: a promising tool for detection of lung cancer. Cancers. 2010;2:32-42.

25. Mazzone PJ. Exhaled breath volatile organic compound biomarkers in lung cancer. J Breath Res. 2012;6:027106.

26. Amann A, Corradi M, Mazzone P, Mutti A. Lung cancer biomarkers in exhaled breath. Expert Rev Mol Diagn. 2011;11:207-17. 
27. Fu XA, Li M, Knipp RJ, Nantz MH, Bousamra M. Noninvasive detection of lung cancer using exhaled breath. Cancer Med. 2014:3:174-81.

28. Fu XA, Li M, Biswas S, Nantz MH, Higashi RM. A novel microreactor approach for analysis of ketones and aldehydes in breath. Analyst. 2011;136:4662-6.

29. Li M, Biswas S, Nantz MH, Higashi RM, Fu XA. Preconcentration and analysis of trace volatile carbonyl compounds. Anal Chem. 2012;84:1288-93.

30. Broza YY, Kremer R, Tisch U, Gevorkyan A, Shiban A, Best LA, et al. A nanomaterial-based breath test for short-term follow-up after lung tumor resection. Nanomedicine. 2013;9:15-21.

31. Horvath I, Lazar Z, Gyulai N, Kollai M, Losonczy G. Exhaled biomarkers in lung cancer. Eur Respir J. 2009;34:261-75.

32. Ligor M, Ligor T, Bajtarevic A, Ager C, Pienz M, Klieber M, et al. Determination of volatile organic compounds in exhaled breath of patients with lung cancer using solid phase microextraction and gas chromatography mass spectrometry. Clin Chem Lab Med. 2009;47:550-60.

\section{Discussion}

Dr Chukwumere Nwogu (Buffalo, NY). Dr Bousamra, I congratulate you and your colleagues for this really intriguing study and your excellent presentation.

With the anticipated marked increase in the number of small lung nodules that we will probably need to evaluate as CT scan screening for lung cancer gets really deployed in the community, I think it is beneficial to have cost-effective tools to refine our management algorithms and to potentially avoid unnecessary or even harmful procedures. I think this is very timely. I know it is early in its development, but I find it very attractive. I also thank you for providing the manuscript ahead of time.

I have 3 questions for you. In your estimation, what do you think the estimated cost of this breath analysis would be for an individual patient and how reproducible is it in various laboratories?

Dr Bousamra. The cost of doing the test right now is about $\$ 45$. There is the mass spectrometry charge, fee. The silicone wafers cost a couple of dollars apiece. The chemical coating is very inexpensive. Then you would have to hire somebody to do the mass spectrometry analysis if this was going to be a system-wide test. I would estimate that the cost to an insurer would probably be $\$ 200$. We have not yet demonstrated reproducibility at other institutions.

The next thing we want to do is enroll 2 or 3 times as many patients in the next year to confirm the results that we have already have or to modify them. Quite frankly, when you begin something, you are not quite as standardized as you should be because you are figuring out how to do it. I think we could be more quantitative than we were to begin with about collecting breath. Why did we miss some of those patients? I did not have time to say it, but of the 12 misses in early-stage disease, 10 of them were adenocarcinoma. So it may be that we are missing indolent tumors. They were small adenocarcinomas, well differentiated for the most part. But it may also be that the patients just did not give us a single exhaled breath. It may be that they cheated and breathed a couple of times, so that most of the breath in the bag was dead-space ventilation.

Dr Nwogu. You suggested using this to assess a patient with bulky disease and maybe decide not to perform a biopsy on such a patient. In my mind, it is more attractive to evaluate small lesions, especially screen-detected lesions. You looked at 37 patients who had stage 0 or stage I tumors that were less than $3 \mathrm{~cm}$. You quoted some values for the sensitivity of your breath analysis versus PET scan, but in that particular subset of patients, what was the specificity of the analysis?

Dr Bousamra. Well, the specificity is the same. It is still dependent on the number of carbonyls that you choose to be positive. If we define 2 or more carbonyl compounds as being positive, for those small lesions we have a sensitivity of $73 \%$ and a specificity of about $80 \%$. In my thinking on helping to avoid intervention in benign diseases, I agree with you, it is not the primary problem. The big factor is that we have got to study or see how we do with $1-\mathrm{cm}, 1.5-\mathrm{cm}, 0.7-\mathrm{cm}$ lesions that are detected on a screening CT scan. That is the elephant in the room. But, oh, by the way, the test never misses bigger tumors, at least in our experience. If the tumor was bigger than $3 \mathrm{~cm}$, breath analysis was never wrong. Say a patient has a $3-\mathrm{cm}$ hilar lesion and you think it is histoplasmosis, but it is active on PET and everybody is worried to death about it, but if the patient blows a stonecold negative breath analysis, it really probably is negative, and that can be reassuring. You are not going to perform a biopsy on it 3 or 4 times. I am not saying that I am going to do that tomorrow, but maybe if the data are confirmed in a larger study, it could be helpful and reassuring to the patient and to the clinician.

Dr Nwogu. Have you considered combining the findings from the PET and your breath analysis? For instance, if you combine the 2 studies, the 2 tests, on a single patient, would you be able to increase the sensitivity and specificity even further?

Dr Bousamra. No. We looked at that, and it did not really work out that way. I threw out a slide that looked at the benign patients and where they were false-negative and false-positive. They migrate together to a fair extent. I do not have enough benign patients to really answer that confidently, but so far they have not been helpful in combination.

Dr Akif Turna (Istanbul, Turkey). Did you look at the diabetes status of the patients and the consumption of alcohol in these 2 groups, especially 3 to 5 hours before the test, and the glucose level of the patients? That may affect the ketone bodies and the aldehydes in the blood.

Dr Bousamra. We did record what their diet was. Most of the tests were taken early in the morning, so I hope they were not drinking too much in the few hours before. We did not specifically record alcohol intake. The point about these compounds is that they are very specific to cancer. We do not find alcohol-related compounds in increased concentrations. The glucose in the diabetics, they had higher levels of acetate, but not the carbonyls we were interested in.

Dr Daniel Miller (Marietta, Ga). Excellent presentation.

I have 2 questions. Was there a difference with regard to histology -adenocarcinoma versus squamous? The second question, for your healthy baseline individuals, did you study any patients with head and neck cancer? I think this would be important because of the relationship between aerodigestive cancers and smoking; your test could be positive for a head and neck cancer that was undetected instead of a lung cancer.

Dr Bousamra. To answer the second question, yes, we have a few head and neck cancers that I did not present and they were positive. We also have a variety of other cancers that are metastatic to the lung that were positive on exhaled breath.

What was the first question? 
Dr Miller. Was there a difference between adenocarcinoma and squamous?

Dr Bousamra. There is a cancer marker profile that is specific for small cell lung cancer. Pentanone and $n$-pentanal are more diagnostic of small cell tumors. But I do not know whether that is a tumor volume relationship. Also, 2-butanone is higher for stages beyond stage I, as we noticed a difference between those 2 . We did not find any strong correlation differentiating between adenocarcinoma and squamous cell carcinoma.
Dr Miller. Can you do this test with urine?

Dr Bousamra. Well, Linus Pauling did.

Dr Miller. Smell it, sniff it? Bring a dog in here and we will let it smell your urine. (Laughter.)

Dr Bousamra. It is a good question.

Dr Miller. But you cannot fake a urine test. Well, some athletes can, but most of the time a patient will not fake a urine test.

Dr Bousamra. It is a good question and we should look at whether a combined analysis would be beneficial. 